

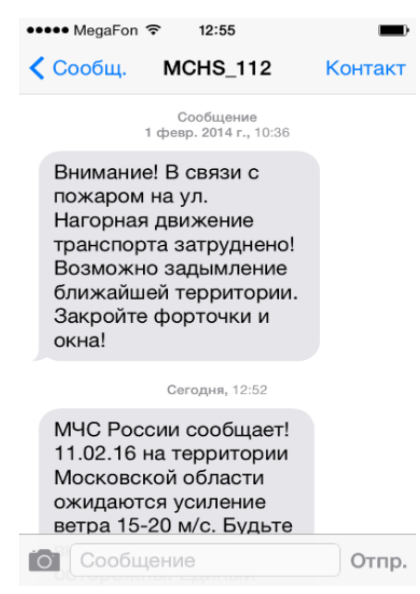

Figure 1. Message from the Russian Ministry of Emergency Situations Sent to Mobile Users Using Geo Targeting

\section{THE NEW APPROACH}

Collection of contact information from sales classified ads posted on the Internet by users is a new efficient way of gathering information about mobile geo targeting. In Russia, Avito.ru is currently the most visited and fastest growing sales site. Daily traffic to this site reaches over 8 million visitors. According to statistics, the site contains more than 33 million ads at the same time. To register on the site and be able to post ads, the user must enter his mobile phone number. This number must be confirmed through a code sent via SMS. The site administration can contact the author of any ad through the confirmed phone number. So, this number has to be current. Apart from having a phone number, each ad contains the author's contact information, including geographical reference (district of the city or metro station). It is most likely that the district indicated by the user is the place where he spends most of his time, such as his residence or place of work. Since most of the sellers are interested in selling their goods and services, it can be argued that apart from their phone numbers, they also provide current contact details. Based on the available data, it can be concluded that advertising site avito.ru contains ads with correct and current mobile phone numbers with tied to a location within the city.

As a new user on the site, when you want to search for ads, you will need to select the appropriate region of the country. Optionally, the site provides you the opportunity to select an area of the city, subway station and product category. Detailed search is performed through selection of product categories or searching by add title. To collect mobile phone numbers tied to a certain metro station, the most optimal is to search for ads based on this metro station without any additional parameters. For example, a search within Moscow and Prospekt Vernadskogo metro station will give you a list of 248,842 ads. The selected metro station serves as the information about the current location, while the cell phone number and name of the owner of the number are contained in the ad. So you will need to get the contents of the page of each ad.

The name of the ad author is contained in the source code of the page in pure form and so does not require further action to collect. The more difficult process is in obtaining the phone number. To read the cell phone number of a seller, you will need to click a special link called "Show phone". This was done by the site to enable it gather statistics on the number of people that views the phone number and to make more complex epy automatic scanners that gather the contact details of ad owners.

In the desktop version of the site, on an ad page, when you click on "Show phone", a image displaying the phone number loads (Figure 2). The image is loaded via a link that looks as follows "https://www.avito.ru/items/phone/ $\{\mathrm{id}\}$ ?pkey=\{pkey $\}$

Where id is the ad number in avito, pkey is the key needed to obtain the image containing the phone number. This link is not in an open form. That is, when you click on the link "show phone", the event captures special function Javascript. This function uses the ad id and a unique hash code specified in the form of a variable. Using these data, the function generates a pkey key using a special algorithm. The function sends a GET request via the specified link, substituting pkey. The result, which is in the form of an image containing the phone number, is placed instead of the "show phone" link. 


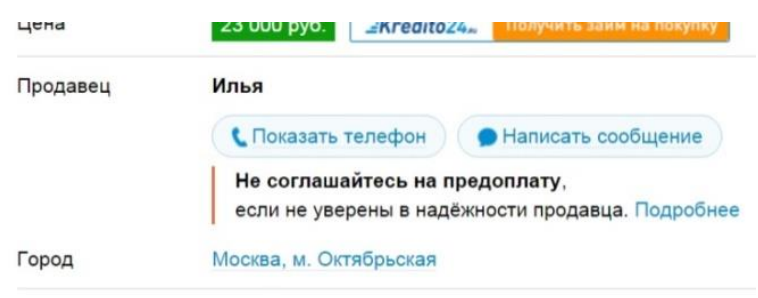

Figure 2. The "Show Phone" Button

The Javascript code that generates pkey and receives the image containing the phone number is obfuscated and hidden in javascript codes to reduce the probability of data collectors reading the number-you require a high level of programming skills to automatically obtain an image containing a phone number.

To automatically receive images containing phone numbers, you need to load the ad page and then extract from its source code the javascript variable value with unique hash code and ad id. Then the pkey generation algorithm needs to be executed using hash code and ad id (Figure 3). After generating the pkey key, you now have all the data needed to obtain the image bearing the phone number. To do this, you need to send a GET request, and while doing that, it is important to specify the appropriate HTTP headers and referrer field in which the ad page URL will be entered. Server response to the GET request with the correct pkey key comes in the form of a data array encoded using base64 algorithm. Data decoding allows to obtain an image bearing the phone number. To save the phone number in the database, the phone number needs to be recognized from the image using a text recognition algorithm.

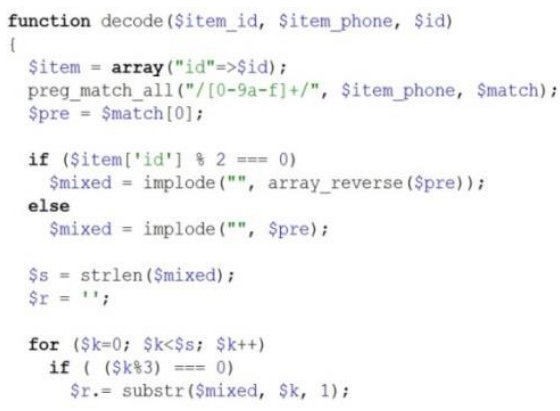

Figure 3. Pkey Generation Process

Apart from the desktop version of the site, the phone number of the ad owner can also be through the mobile version of the site. A link to the mobile version differs by how the subdomain of the site is written: the desktop version uses the domain "www.avito.ru", while the mobile version goes with "m.avito.ru". The other parameters in the ad links are identical to each other. The peculiarities of the mobile version simplify the task of collecting phone numbers because the numbers are loaded as text without images. This is done for the convenience of mobile users - writing of phone numbers in the original form by text allows you to call that mobile device with a single click. If the phone number was to be transmitted in the form of an image, users would find it hard to dial the number manually.

To receive a phone number on the mobile version of the site, a link needs to be read from the ad page code. The link takes the form http://\{url\}/phone/\{hash\}? async.

Where url is the full url of the ad, hash is the hash number indicated in the link. After the link has been created, you then need to compose and send a GET request with the correct headers and referrer field, which contains the ad URL. The server response will contain a data array containing the phone number in an open form.

To collect data from ad pages in large amount and within a limited period of time, it is necessary to simulate different users by sending requests from different IP addresses. Otherwise, the avito server may block the IP address. If the data collection time is not limited, data can be collected from a small number of IP addresses, but maintaining more than 1 second interval between requests with clearing of cookies files.

Ad details are stored in a database table. The table has a number of fields, each of which stores certain data from the ad, such as Name, phone number, ID of the metro station or district, collection date, 
title, link to the ad (Figure 4). To implement simple mailing, the system only needs to select mobile users from the database for a particular area. Including users' names in the text messages potentially enhances the efficiency of the mailing. Data from ad titles can be used to select users of specialized niches, for example, fans of winter fishing.

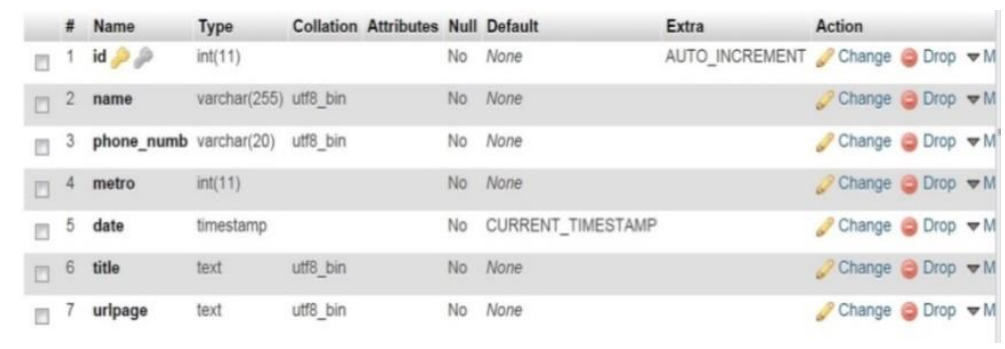

Figure 4. Data Storage in the MYSQL-Table

\section{CONCLUSION}

Geo targeting of mobile phone numbers is not available in any form. One cannot determine the location of a mobile phone number. Using a popular add site, you can collect and daily update the database of cell phone numbers with geo targeting in areas within the city. This makes it possible to efficiently inform and notify mobile users in certain districts of the city through SMS text messages and calls. This in turn reduces the cost of providing information and makes advertising more targeted, thereby strengthening customer loyalty. If you use geo targeting in conjunction with ad category information, you can determine the approximate interests and hobbies of subscribers. This would enable you send texts only to potentially interested mobile users and reduce the likelihood of users reacting negatively to the SMS advertising. With information about categories of ads without precise geo targeting, you will be able to provide relevant information and notification to interested mobile users within the city.

\section{REFERENCES}

[1] P. Ngo and D. Wijesekera, "Enhancing the Usability of the Commercial Mobile Alert System," In Critical Infrastructure Protection V, Springer Berlin Heidelberg, pp. 137-149, 2011.

[2] S. Heath, "U.S. Patent Application,” No. 13/337, pp. 275, 2011.

[3] Lyamin A. V., et al., "Circadian Rhythms, Effects of Their Changes, and Asthenia in the Context of Higher Educational Studies with Remote Learning," Neuroscience and Behavioral Physiology, vol/issue: 45(5), pp. 583$585,2015$.

[4] T. S. Krivonogova, et al., "Effect of respiratory and aqua-gymnastics on the adaptive potential of pregnant women," Voprosy kurortologii, fizioterapii, i lechebnoǐ fizicheskǒ kultury, vol. 6, pp. 25-29, 2010.

[5] L. Lisitsyna, et al., "Estimation of Student Functional State in Learning Management System by Heart Rate Variability Method," Smart Digital Futures, IOS Press, vol. 262, pp. 726-731, 2014.

\section{BIOGRAPHY OF AUTHOR}

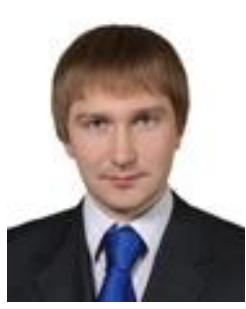

Assosiated Professor at Department of Information Systems and Technologies, National Research Nuclear University MEPhI (Moscow Engineering Physics Institute). Doing researches in the field of information secutiry. 